\title{
Suitability of the Montreal Cognitive Assessment versus the Mini-Mental State Examination in Detecting Vascular Cognitive Impairment.
}

\section{$\operatorname{AUTHOR(S):~}$}

Ihara, Masafumi; Okamoto, Yoko; Takahashi, Ryosuke

\section{CITATION:}

Ihara, Masafumi ...[et al]. Suitability of the Montreal Cognitive Assessment versus the MiniMental State Examination in Detecting Vascular Cognitive Impairment.. Journal of stroke and cerebrovascular diseases 2013, 22(6): 737-741

\section{ISSUE DATE:}

2013-08

URL:

http://hdl.handle.net/2433/177914

\section{RIGHT:}

(C) 2013 National Stroke Association. Published by Elsevier Inc.; This is not the published version. Please cite only the published version.; この 論文は出版社版でありません。引用の際には出版社版をご確認ご利用 ください。 


\title{
Suitability of the Montreal Cognitive Assessment versus the Mini-Mental State Examination in detecting vascular cognitive impairment
}

\author{
Masafumi Ihara*, Yoko Okamoto, Ryosuke Takahashi \\ Department of Neurology, Kyoto University Graduate School of Medicine \\ 54 Kawahara-cho, Shogoin, Sakyo, Kyoto 606-8507 Japan
}

*Correspondence should be addressed:

Masafumi Ihara, MD, PhD, FACP

Department of Neurology, Kyoto University Graduate School of Medicine

54 Kawahara-cho, Shogoin, Sakyo, Kyoto 606-8507 Japan

$\mathrm{Tel} / \mathrm{fax}+81-757513766$

E-mail: ihara@kuhp.kyoto-u.ac.jp

Short title: MoCA-J for vascular cognitive impairment

Author's contribution:

Masafumi Ihara; patient care, neuropsychological assessment, statistical analysis

Yoko Okamoto; neuropsychological assessment

Ryosuke Takahashi; study supervision 


\section{Abstract}

Goal:

The Mini-Mental State Examination (MMSE) has been criticized as an insufficient screening test for patients with vascular cognitive impairment due to insensitivity to visuospatial and executive functional deficits. The Montreal Cognitive Assessment (MoCA) was designed to be more sensitive to such deficits and may therefore be a superior screening instrument for vascular cognitive impairment.

Methods:

Twelve patients with extensive leukoaraiosis on magnetic resonance imaging ( $76.0 \pm 8.7 \mathrm{yrs}$ on average) underwent neurological and cognitive measures, including the Japanese version of MoCA (MoCA-J) and MMSE. Accepted cutoffs of MMSE $<27$ and MoCA $<26$ were taken to indicate cognitive impairment. $Z$ scores were calculated to evaluate the discriminating ability of individual MMSE and MoCA-J subtest scores.

Findings:

Although there was a strong correlation between the total MoCA-J and MMSE scores $(r=0.90, p<0.0001)$, MMSE scores were skewed toward the higher scores (range 18-30, median 28), while MoCA-J scores 
were normally distributed (range 9-28, median 21). Of the seven patients with unimpaired MMSE scores,

six $(86 \%)$ patients had an impaired MoCA-J score. Z scores were $>5$ for four MMSE subtests (orientation,

registration, naming, and language) but for only one MoCA-J subtest (naming).

Conclusions:

The MoCA-J better discriminated cognitive status between subjects with extensive leukoaraiosis.

MoCA-J is more sensitive than the MMSE in screening for cognitive impairment in patients with subcortical vascular cognitive impairment.

Keywords: leukoaraiosis, Montreal Cognitive Assessment, screening, stroke, vascular cognitive impairment 


\section{Introduction}

Cognitive impairment resulting from cerebrovascular insufficiency has been termed vascular cognitive

impairment (VCI). ${ }^{1} \mathrm{VCI}$ forms a spectrum that includes mild cognitive impairment of vascular etiology (vascular MCI), vascular dementia, mixed Alzheimer's disease with a vascular component. ${ }^{2,3}$ As vascular MCI patients with more severe impairment were found to be at higher risk of conversion to dementia, compared to patients with less severe or no cognitive impairment, ${ }^{4}$ early detection of cognitive deficits may facilitate in preventing cognitive deterioration.

The widely used Mini-Mental State Examination (MMSE) ${ }^{5}$ was found to be inaccurate in screening VCI as it was insensitive to MCI or complex cognitive deficits specifically. ${ }^{1,6}$ By comparison, the Montreal Cognitive Assessment (MoCA) has been designed to be sensitive to mild deficits ${ }^{7}$ and has been evaluated in amnestic $\mathrm{MCI}^{7}$ and Parkinson disease ${ }^{8}$. Importantly, MoCA is reported to detect more cognitive abnormalities after ischemic stroke or transient ischemic attack, compared to MMSE, particularly in relation to executive function, attention and delayed recall. ${ }^{9,}{ }^{10}$ In addition, the Hong Kong MoCA is reported to be useful for use in Chinese patients with leukoaraiosis; ${ }^{11}$ however, direct comparison of MoCA with MMSE scores has not been performed in VCI. 
Hence, the primary aim of the present study was to test the hypothesis that MoCA is more sensitive than

MMSE in the detection of cognitive impairment in patients with ischemic leukoaraiosis, namely

subcortical VCI, which incorporates two clinical entities 'Binswanger's disease' and 'the lacunar state'.

The feasibility and the psychometric properties of MoCA (modified for Japanese population; MoCA-J) were compared with MMSE.

\section{Methods}

Subjects

Twelve patients whose FLAIR- and T2-weighted MRI scans revealed confluent hyperintensities in the

subcortical white matter (Schmidt scale score of 3$)^{12}$ and several punctate high-intensity areas in the basal ganglia were studied. The patients were enrolled because of the above neuroradiological abnormalities after visiting our Neurology Clinic from May 2008 to March 2011 with various neurologic signs and symptoms. Patients with mild to moderate leukoaraiosis (Schmidt scale score of 1 or 2) were not enrolled in this study to exclude the possibility of leukoaraiosis associated with neurodegenerative disorders, such as Alzheimer's disease. Each subject was fully instructed on the experimental procedures and provided with written, informed consent, as approved by the Committee of Medical Ethics within our faculty. None 
of the patients had apparent lesions in the cerebral cortex or hippocampus. MR angiography and duplex

color-coded sonography did not reveal $>50 \%$ stenosis in the major intracranial and extracranial vessels.

Neuropsychological evaluation

All subjects underwent a general physical and neurologic examination and neuropsychological

assessment, including the MoCA-J and MMSE. To avoid habituation and interference effects, MMSE was

carried out at least one week after MoCA-J assessment. Two neurologists were involved in the

neuropsychological assessment; if their assessments did not correlate, patients were reexamined by the

team for a final verdict. A cutoff of $\geqq 27$ on MMSE score was chosen to indicate normal cognitive

function ${ }^{13}$ and the accepted cutoff of $<26$ on the MoCA-J was taken to indicate cognitive impairment. ${ }^{14}$

The $\mathrm{Z}$ score analysis

For both the MMSE and MoCA-J subtests, the $\mathrm{Z}$ scores were calculated by dividing the mean subtest

score by its standard deviation, where lower $\mathrm{Z}$ scores indicate greater discrimination between subjects.

Statistical analysis

The statistical significance of intergroup differences was assessed with Fisher's exact test for categorical variables, and continuous variables of demographic data by the Mann-Whitney U test. Possible links 
between the variables were observed using Pearson correlation analysis.

\section{Results}

Demographic data of the patients

Patient clinical features and demographics are summarized in the Table 1. All patients had at least 1 risk factor for ischemic cerebrovascular disease, including hypertension and diabetes mellitus. Eight of 12 patients (67\%) showed gait disturbance, the most frequent neurological deficit. The mean modified Rankin Scale score was 1.4 (range, 1-2). Most of the patients showed lacunar infarctions or microbleeds in the basal ganglia or the thalamus as a manifestation of underlying small vessel pathology. Their clinical histories and radiological features excluded possibility of co-existing single-strategic infarct dementia.

Relationship between MoCA-J and MMSE scores

There was a significant relationship between the MoCA-J and MMSE scores $(r=0.90, p<0.0001)$. MMSE scores were skewed toward the higher scores (range 18-30, median 28) while MoCA-J scores were normally distributed (range 9-28, median 21) (Figure 1). Of the 11 patients with impaired MoCA-J scores, only five (45\%) patients had an impaired MMSE score, while all 11 patients with impaired MMSE scores 
had an impaired MoCA-J score. Of the seven patients with unimpaired MMSE scores, six (86\%) had an impaired MoCA-J score.

Z score analysis

Individual subtests of the MMSE and MoCA-J, together with the results, are described in Table 2 and

Table 3, respectively. Z scores were >5 for four MMSE subtests (orientation, registration, naming, and language) but only for one MoCA-J subtest (naming).

Clinical utility of the MoCA-J

The MoCA-J was accomplished by all the Japanese elderly subjects with VCI enrolled in this study. The average administration time was 12 minutes.

Correlation analysis between the MoCA-J score and the MRI-based cerebrovascular lesions

The MoCA-J score was significantly correlated with the number of lacunar infarctions $(r=-0.67, p=0.03)$ but not with the number of microbleeds in the basal ganglia or the thalamus $(r=-0.39, p=0.28)$.

\section{Discussion}


The main finding of this study is that MoCA-J is more sensitive than the MMSE in detecting subcortical

VCI. Eleven of the 12 patients with either impaired MoCA-J or MMSE scores could only be detected by impaired MoCA-J scores, while none of the patients presented with only impaired MMSE scores. In addition, the $\mathrm{Z}$ score analysis suggested that the MoCA-J better discriminated cognitive status between subjects. Therefore, MoCA-J could be a useful instrument to detect VCI at an earlier stage in patients with extensive leukoaraiosis. The significant correlation of the MoCA-J score with the number of lacunar infarctions further suggests the clinical relevance of this screening test in the detection of VCI characterized by small vessel pathology. It took only 12 minutes to administer the MoCA-J even in subcortical VCI patients.

The poorer performance of the MMSE in detecting subcortical VCI may be due to several factors. The

MMSE is less sensitive in detecting impairment in visuospatial or executive function, characteristic features that are preferentially impaired in $\mathrm{VCI}^{2,15}$ In light of such restrictions of MMSE, the trail-making test or digit symbol test has been recommended to supplement the MMSE to improve its sensitivity in the cognitive assessment of subjects with leukoaraiosis. ${ }^{16}$ Furthermore, visuo-executive dysfunction has been found to predict poor survival after stroke; ${ }^{17}$ therefore, early detection of VCI with MoCA is ideal for earlier initiation of intervention and improved outcome. Vascular risk factors should be adequately controlled to achieve a reduction in the risk of dementia. ${ }^{18}$ 
The MoCA-J demonstrated deficits in other cognitive domains, such as recall and language. As the specific assessment criteria to test recall and language are less complicated in MMSE than in MoCA-J, the ceiling effect may prevent MMSE from detecting intersubject differences in cognitive impairment. In support of this, the $\mathrm{Z}$ scores for recall and language in MoCA-J were lower than those in MMSE, indicating higher discriminating power of MoCA-J in assessment of cognitive impairment. Recently, vascular risk factors have been reported to promote conversion from MCI to Alzheimer's disease. ${ }^{19}$ Moreover, in patients who have clinically diagnosed Alzheimer's disease, treatment of vascular risk factors is associated with a slower decline in MMSE score. ${ }^{20} \mathrm{~A}$ recent animal study has suggested that the effect of Alzheimer's neurodegeneration and VCI are not only additive but also synergistic in worsening cognitive impairment. ${ }^{21}$ Therefore, the early diagnosis of amnestic MCI with $\mathrm{MoCA}^{7,14}$ may also benefit patients by the introduction of optimal control of any vascular risk factors, which may prevent conversion of MCI to dementia.

The main limitation is a small number of patients enrolled in this study as only those with most severe white matter changes were eligible for this study to minimize heterogeneity of VCI and to exclude those with white matter changes secondary to neurodegenerative changes to the utmost extent. ${ }^{22}$ This 
preliminary study should therefore be viewed as hypothesis generating and should be followed by larger studies to confirm the results. Nevertheless, our study provided a direct comparison of MMSE and MoCA-J, which showed an appreciably greater suitability of the MoCA-J against the MMSE in the detection of VCI.

In conclusion, the MoCA-J is a promising tool for the screening for cognitive impairment in VCI patients with leukoaraiosis as it may detect complex cognitive impairments, such as in executive function and visual perception, at earlier stages of VCI. 


\section{Acknowledgments}

We are very grateful to Dr Ahmad Khundakar for editing of this manuscript, and to Ms Maya Uose for her secretarial work.

\section{Disclosure}

This work was supported in part by grants from the Japan Health Foundation, the Mitsui Sumitomo

Insurance Welfare Foundation, and the Univers Foundation (M.I.). 


\section{References}

1 Hachinski V, Iadecola C, Petersen RC, et al. National Institute of Neurological Disorders and Stroke-Canadian Stroke Network vascular cognitive impairment harmonization standards. Stroke 2006; 37: 2220-2241. O'Brien JT, Erkinjuntti T, Reisberg B, et al. Vascular cognitive impairment. Lancet Neurol 2003; 2: 89-98.

3 Rockwood K, Wentzel C, Hachinski V, et al. Prevalence and outcomes of vascular cognitive impairment. Vascular Cognitive Impairment Investigators of the Canadian Study of Health and Aging. Neurology 2000; 54: 447-451. Narasimhalu K, Ang S, De Silva DA, et al. Severity of CIND and MCI predict incidence of dementia in an ischemic stroke cohort. Neurology 2009; 73: 1866-1872. Folstein MF, Folstein SE, McHugh PR. "Mini-mental state". A practical method for grading the cognitive state of patients for the clinician. J Psychiatr Res 1975; 12: 189-198. Nys GM, van Zandvoort MJ, de Kort PL, et al. Restrictions of the Mini-Mental State Examination in acute stroke. Arch Clin Neuropsychol 2005; 20: 623-629. Nasreddine ZS, Phillips NA, Bedirian V, et al. The Montreal Cognitive Assessment, MoCA: a brief screening tool for mild cognitive impairment. J Am Geriatr Soc 2005; 53: 695-699.

Zadikoff C, Fox SH, Tang-Wai DF, et al. A comparison of the mini mental state exam to the Montreal cognitive assessment in identifying cognitive deficits in Parkinson's disease. Mov Disord 2008; 23: 297-299.

9 Pendlebury ST, Cuthbertson FC, Welch SJ, et al. Underestimation of cognitive impairment by mini-mental state examination versus the montreal cognitive assessment in patients with transient ischemic attack and stroke: a population-based study. Stroke 2010; 41: 1290-1293.

10 Dong Y, Sharma VK, Chan BP, et al. The Montreal Cognitive Assessment (MoCA) is superior to the Mini-Mental State Examination (MMSE) for the detection of vascular cognitive impairment after acute stroke. J Neurol Sci 2010; 299: 15-18.

11 Wong A, Xiong YY, Kwan PW, et al. The validity, reliability and clinical utility of the Hong Kong Montreal Cognitive Assessment (HK-MoCA) in patients with cerebral small vessel disease. Dement Geriatr Cogn Disord 2009; 28: 81-87.

12 Schmidt R, Hayn M, Fazekas F, et al. Magnetic resonance imaging white matter 
hyperintensities in clinically normal elderly individuals. Correlations with plasma concentrations of naturally occurring antioxidants. Stroke 1996; 27: 2043-2047.

13 Crum RM, Anthony JC, Bassett SS, et al. Population-based norms for the Mini-Mental State Examination by age and educational level. JAMA 1993; 269: 2386-2391.

14 Fujiwara Y, Suzuki H, Yasunaga M, et al. Brief screening tool for mild cognitive impairment in older Japanese: validation of the Japanese version of the Montreal Cognitive Assessment. Geriatr Gerontol Int 2010; 10: 225-232.

15 Ihara M, Tomimoto H, Ishizu K, et al. Decrease in cortical benzodiazepine receptors in symptomatic patients with leukoaraiosis: a positron emission tomography study. Stroke 2004; 35: 942-947.

16 O'Sullivan M, Morris RG, Markus HS. Brief cognitive assessment for patients with cerebral small vessel disease. J Neurol Neurosurg Psychiatry 2005; 76: 1140-1145.

17 Oksala NK, Jokinen H, Melkas S, et al. Cognitive impairment predicts poststroke death in long-term follow-up. J Neurol Neurosurg Psychiatry 2009; 80: 1230-1235.

18 Di Legge S, Hachinski V. Prospects for prevention and treatment of vascular cognitive impairment. Curr Opin Investig Drugs 2003; 4: 1082-1087.

19 Li J, Wang YJ, Zhang M, et al. Vascular risk factors promote conversion from mild cognitive impairment to Alzheimer disease. Neurology 2011; 76: 1485-1491.

20 Deschaintre Y, Richard F, Leys D, et al. Treatment of vascular risk factors is associated with slower decline in Alzheimer disease. Neurology 2009; 73: 674-680.

21 Yamada M, Ihara M, Okamoto Y, et al. The Influence of Chronic Cerebral Hypoperfusion on Cognitive Function and Amyloid beta Metabolism in APP Overexpressing Mice. PLoS ONE 2011; 6: e16567.

22 Ihara M, Polvikoski TM, Hall R, et al. Quantification of myelin loss in frontal lobe white matter in vascular dementia, Alzheimer's disease, and dementia with Lewy bodies. Acta Neuropathol 2010; 119: 579-589. 
Table 1. Patient clinical features and demographics

\section{Variable}

Mean age (range), y

$76 \pm 8.7(55-84)$

Gender

M, 7; F, 5

Modified Rankin Scale, mean score (range)

$1.4 \pm 0.5(1-2)$

Gait disturbance, No. (\%)

$8(67)$

Dysarthria, No. (\%)

$4(33)$

Urinary incontinence, No. (\%)

$3(25)$

Hypertension, No. (\%)

$7(58)$

Diabetes mellitus, No. (\%)

$3(25)$

Cigarette smoking, No. (\%)

$3(25)$

Sleep apnea, No. (\%)

$2(17)$

Previous CVD, No. (\%)

$6(50)$

Use of antithrombotics, No. (\%)

$8(67)$

Use of statins, No. (\%)

$6(50)$

LDL Cholesterol, mg/dL

$115 \pm 44$

Triglyceride, $\mathrm{mg} / \mathrm{dL}$

$112 \pm 45$ 
Hematocrit, \%

Fibrinogen, mg/dL

$1.5 \pm 2.0$

$286 \pm 53$
$39.6 \pm 3.9$

No. of lacunas in the BG/thalamus

$2.9 \pm 2.8$

BG, basal ganglia; CVD, cerebrovascular disease; MBs, microbleeds 
Table 2. Mean of MoCA-J subtest scores with different cognitive screening test results

\begin{tabular}{|c|c|c|c|c|c|c|c|}
\hline MOCA-J & $\begin{array}{c}\text { Visuo- } \\
\text { executive/5 }\end{array}$ & Naming/3 & Attention/6 & Language/3 & Abstraction/2 & Recall/5 & Orientation/6 \\
\hline Pt 1 & 1 & 2 & 4 & 1 & 1 & 0 & 5 \\
\hline Pt 2 & 1 & 3 & 3 & 0 & 0 & 0 & 2 \\
\hline Pt 3 & 3 & 3 & 6 & 1 & 2 & 3 & 6 \\
\hline $\mathrm{Pt} 4$ & 2 & 3 & 3 & 1 & 2 & 0 & 4 \\
\hline Pt 5 & 3 & 2 & 6 & 2 & 1 & 4 & 5 \\
\hline Pt 6 & 4 & 3 & 5 & 2 & 2 & 3 & 6 \\
\hline $\mathrm{Pt} 7$ & 3 & 3 & 6 & 1 & 1 & 2 & 6 \\
\hline Pt 8 & 4 & 3 & 5 & 1 & 1 & 0 & 6 \\
\hline Pt 9 & 2 & 3 & 6 & 2 & 2 & 0 & 6 \\
\hline Pt 10 & 5 & 3 & 6 & 2 & 2 & 4 & 6 \\
\hline Pt 11 & 3 & 3 & 5 & 1 & 0 & 0 & 5 \\
\hline Pt 12 & 2 & 3 & 6 & 2 & 2 & 0 & 4 \\
\hline Average & 2.8 & 2.8 & 5.1 & 1.3 & 1.3 & 1.3 & 5.1 \\
\hline SD & 1.2 & 0.4 & 1.2 & 0.7 & 0.8 & 1.7 & 1.2 \\
\hline Z score & 2.3 & 7.3 & 4.4 & 2.0 & 1.7 & 0.8 & 4.1 \\
\hline
\end{tabular}


MoCA-J domain subtest maximum score/details: visuo-executive/5 - trail B test, cube copy, clock

drawing; attention/6 - digit span, vigilance (tapping at the Hiragana 'a' in a list of Hiragana characters),

serials $7 \mathrm{~s}$; recall $/ 5$ - recall a list of 5 words; orientation $/ 6$ - date, month, year, day, place, city;

abstraction/2 - similarities between two items; language/ 3 - sentence repetition, verbal fluency; naming/3

- confrontation naming (lion, rhino, camel). 
Table 3. Mean of MMSE subtest scores with different cognitive screening test results

\begin{tabular}{|c|c|c|c|c|c|c|c|}
\hline MMSE & Orientation/10 & Registration/3 & $\begin{array}{c}\text { Attention/ } \\
\text { Calculation/5 }\end{array}$ & Recall/3 & Naming/2 & Language/6 & Drawing/1 \\
\hline Pt 1 & 9 & 3 & 4 & 2 & 2 & 3 & 0 \\
\hline Pt 2 & 5 & 2 & 4 & 0 & 1 & 5 & 1 \\
\hline Pt 3 & 10 & 3 & 5 & 3 & 2 & 6 & 1 \\
\hline Pt 4 & 10 & 3 & 1 & 0 & 2 & 5 & 1 \\
\hline $\operatorname{Pt} 5$ & 10 & 3 & 4 & 2 & 2 & 5 & 1 \\
\hline Pt 6 & 10 & 3 & 5 & 2 & 2 & 6 & 1 \\
\hline Pt 7 & 10 & 3 & 5 & 3 & 2 & 6 & 1 \\
\hline Pt 8 & 10 & 3 & 4 & 0 & 2 & 6 & 1 \\
\hline Pt 9 & 10 & 3 & 4 & 3 & 2 & 6 & 1 \\
\hline Pt 10 & 10 & 3 & 5 & 3 & 2 & 6 & 1 \\
\hline Pt 11 & 10 & 3 & 1 & 0 & 2 & 6 & 1 \\
\hline Pt 12 & 9 & 3 & 5 & 3 & 2 & 6 & 1 \\
\hline Average & 9.4 & 2.9 & 3.9 & 1.8 & 1.9 & 5.5 & 0.9 \\
\hline SD & 1.4 & 0.3 & 1.4 & 1.4 & 0.3 & 0.9 & 0.3 \\
\hline Z score & 6.5 & 10.1 & 2.7 & 1.3 & 6.6 & 6.1 & 3.2 \\
\hline
\end{tabular}


MMSE domain subtest maximum score / details: attention/calculation/5 - serial $7 \mathrm{~s}$; recall/3 - recall a list of 3 words; orientation/10 - orientation to place and time; naming/2 - confrontation naming (pen, watch); language/ 6 - sentence repetition, comprehension, reading, writing; registration/3 - repeat 3 words; praxis/1 - copy intersecting pentagons. 


\section{Figure legends}

Figure 1. Distribution of MoCA-J and MMSE scores of the patients with extensive leukoaraiosis. Cutoffs

of MMSE <27 and MoCA-J <26 were taken to indicate cognitive impairment (dotted line). 